\title{
Transferencias fonético-fonológicas del español en el mapudungún hablado por la población adulta de Alto Biobío, Octava Región, Chile
}

\author{
Daniela Mena** \\ Gastón Salamanca $^{* * *}$
}

\section{Resumen}

El foco de este artículo son las transferencias fonético-fonológicas del español en el chedungún hablado en Alto Biobío. La muestra está constituida por 30 hablantes adultos, 15 de género masculino y 15 de género femenino. El instrumento utilizado es la lista léxica presente en Croese (1980). Diez fueron las localidades encuestadas: Se analizó un total de 8800 tokens. Se puede concluir lo siguiente: a) una gran vitalidad del chedungún ( $82.67 \%$ de realizaciones indicadoras de vitalidad), y b) el comportamiento de las variables "género" y "subsector" es altamente homogéneo. Es interesante constatar la fuerte resistencia del chedungún al desplazamiento producto del contacto con el español.

Palabras clave: Fonología del mapudungún/chedungún, Transferencias fonéticofonológicas, Vitalidad fonológica.

\section{Phonetic-phonological transfers of Spanish in the Mapudungun spoken by adults in Alto Biobío, Octava Región, Chile}

\begin{abstract}
The focus of this paper are the phonetic-phonological tranfers of Spanish in the Chedungún spoken in Alto Biobío. The sample is made up of 30 adults speakers, 15 male and 15 female. The instrument used is the lexical list present in Croese (1980). Ten were the localities surveyed. We analyzed a total of 8800 tokens. We can conclude the following: a) A great vitality of the Chedungún ( $82.67 \%$ of realizations that indicate vitality), and b) the behavior of the variables "gender" and "subsector" is highly homogenous. It is interesting to note the strong resistance of the Chedungún to the displacement from contact with Spanish.
\end{abstract}

Keywords: Mapudungun/Chedungún Phonology, phonetical-phonological transfers, Phonological vitality.

Recibido: 01/09/17

Aceptado: 03/03/18

Este artículo está asociado al proyecto FONDECYT Regular 1131095 “Adscripción dialectal y reanálisis de aspectos controversiales de la fonología segmental del chedungún hablado en el Alto Biobío”. ** Chilena. Dr. (c) Daniela A. Mena Sanhueza. Universidad de Concepción. Programa de Doctorado en Lingüística. Facultad de Humanidades y Arte, Concepción, Chile. danielamenasanhueza@gmail.com **** Chileno. Dr. Gastón F. Salamanca Gutiérrez. Universidad de Concepción. Departamento de Español. Facultad de Humanidades y Arte, Concepción, Chile. gaston.salamanca@gmail.com 


\section{Introducción}

El mapudungún es la lengua originaria mayoritaria en Chile, y se habla también en Argentina. El número de hablantes en Chile "se encuentra entre unas 140.000 y unas 400.000 personas, dependiendo de qué significa ser hablante y de cuántas personas se autoidentifican como mapuches" (Zúñiga, 2007, p. 46). Estos se concentran fundamentalmente entre la VIII y X regiones de este país, aunque hay una cantidad importante que ha emigrado a la capital, Santiago.

Los fonemas segmentales de esta lengua son los siguientes: vocales/a,

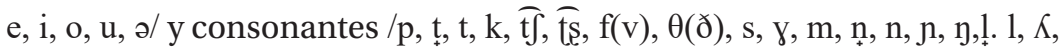
t. jw, $(\delta)$ /. La presencia de 6 vocales fonémicas es reportada portodas las descripciones clásicas de esta lengua. En cuanto a las consonantes, hay una tendencia a la simetría en los inventarios, aunque los fonos fricativo velar [y] y fricativo postalveolar [ $\left.\int\right]$ son segmentos de estatus fonémico controversial.

De acuerdo con Croese (1980), es posible distinguir tres macro grupos dialectales: rama norte (que incluye los grupos I y II), rama central (que incluye los grupos II al VII) y rama sur (que incluye el grupo VIII). Las descripciones fonético-fonológicas clásicas de esta lengua se han focalizado fundamentalmente en la rama central. Este es el caso, por ejemplo, de Suárez (1959), Echeverría (1964), Salas (1976), Lagos (1981), Sadowsky et al. (2013), y Sánchez y Salamanca (2015); por su parte, los trabajos de Sánchez (1989), Salamanca (1997) y Salamanca y Quintrileo (2009) corresponden a la rama norte; mientras que las descripciones de ÁlvarezSantullano (1986) y Sadowsky et al. (2015), corresponden a la rama sur. La mayoría de estas presentaciones utilizan como marco de referencia el distribucionalismo/funcionalismo, tal como se presenta, entre muchos otros, en Trubetzkoy (1987[1939]), Pike (1961[1947]), Martinet (1984) y Burquest (2009[2006]).

Dos rasgos tipológicos que se destacan en las descripciones de las variantes del área norte y central de esta lengua son la ocurrencia de las consonantes interdentales $/ \mathrm{t}, \mathrm{n}, \mathrm{l} /$, opuestas fonológicamente a las alveolares / $\mathrm{t}, \mathrm{n}, \mathrm{l} / \mathrm{y}$ la presencia de 5 segmentos fonológicamente contrastivos en la serie nasal: /m,n, n, n, y/. El grupo VIII presenta solo parcialmente estos rasgos, debido a un proceso de desfonologización sufrido históricamente (Salas, 2006 [1992]: 52). 
Uno de los rasgos dialectales más destacado de esta lengua, de acuerdo con parámetros fonético-fonológicos, es el predominio de las realizaciones sonoras [ð] y [v] en la zona norte; y de sus contrapartes áfonas $[\theta]$ y $[\mathrm{f}]$, en la zona sur.

En relación con la vitalidad del mapudungún, Gundermann et al. (2009:40) señalan que "La distribución del bilingüismo mapuche manifiesta una considerable heterogeneidad. Hay zonas en que el mapuzugun ha desaparecido, en otras se conserva poco y en unas cuantas se mantiene vital".

De acuerdo con el estudio de UTEM-CONADI (2007), en la Región de Los Ríos y Los Lagos un 90,8\% de los mapuches no posee competencia en la lengua; en la Región del Biobío, un 80\%; y en la Región de la Araucanía, un $48,8 \%$ no poseen dicha competencia.

También se ha establecido que la vitalidad del mapudungún es mayor en las zonas rurales que en las zonas urbanas (Gundermann et al, 2009:42). De acuerdo con Lagos (2012: 178), esto puede atribuirse a los siguientes factores:

a) la pérdida de la comunidad tradicional y, con ello, de redes sociales efectivas en las que la lengua se produzca y reproduzca; b) la pérdida de la familia como espacio para aquella función; y c) la pérdida del control cultural sobre la lengua como bien simbólico.

Con respecto a la edad de los hablantes más competentes, el estudio de UTEM-CONADI (2007) estableció que más del 80\% de la población de individuos entre 10 y 30 años no posee competencia en mapudungún. La situación es distinta entre los individuos más adultos, por lo que es posible hablar de una proporción, donde, en términos generales, a mayor edad, mayor es el dominio del mapudungún. En palabras de Gundermann et al. (2009).

La lengua quedó o está cada vez más quedando relegada a las generaciones de mayores y ancianos, sin que se produzca una transmisión extensiva de la misma. Su empleo se limita, la frecuencia de uso se enrarece y las situaciones sociales que la implican son exclusivamente intraétnicas. (p. 43).

Frente a este desalentador panorama, Olate y Henríquez (2010) constatan actitudes positivas entre los profesores de enseñanza básica de colegios insertos en el PEIB (Programa de Educación Intercultural Bilingüe), con respecto a la importancia, valoración y proyección de la 
lengua mapuche. También, en la última década, se ha detectado un proceso de reetnificación que puede redundar en un retardo o reversión del desplazamiento sistemático de la lengua mapuche por parte del español. Tal proceso de reetnificación se puede observar, por ejemplo, en la transición de un bilingüismo pasivo a uno activo y la configuración de nuevos espacios para interactuar en mapudungún, en algunos contextos urbanos (Wittig, 2009).

El marco de referencia para nuestra presentación, con respecto al contacto lingüístico en general y de las transferencias fonético-fonológicas en particular, es aquel que se configura a partir de los aportes de, entre otros, Weinreich (1953), Sala (1988), Blas Arroyo (1991), Silva-Corvalán (2001), Thomason (2001), Medina López (2002), Appel y Muysken (2005),Moreno Fernández (2005), Serrano (2011) y Henríquez (2013). Ellos despliegan temáticas claves, como el contacto lingüístico, definiciones y tipos de bilingüismo, definiciones y tipos de transferencias fonético-fonológicas, etc., todas ellas pertinentes para el trabajo que presentamos.

Para el estudio del contacto mapudungún-español, son centrales las referencias de Salas (1971, 1983), Hernández y Ramos (1978, 1979, 1983, 1984), Sepúlveda (1976), Durán y Ramos (1988a y 1988b), Lagos y Olivera (1988) y Olate y Henríquez (2010). Estos autores se han referido a aspectos del bilingüismo mapudungún-español tales como el castellano mapuchi$z a d o$, la "incidencia del bilingüismo en la aplicación del sistema nacional de educación a la población mapuche infantil" (Salas, 2006 [1992]: 44), el desarrollo de la castellanización en La Araucanía y las actitudes lingüísticas de profesores mapuches de enseñanza primaria, entre otros tópicos.

Henríquez (2004) y Henríquez y Salamanca (2015) se han ocupado del estudio de las transferencias del español en el mapudungún. Hay, sin embargo, algunas diferencias entre estas aproximaciones y las consideraciones metodológicas que se plantean en este artículo (Cf. Consideraciones Metodológicas).

Así, entonces, el planteamiento de estos problemas anticipa una contribución importante tanto al conocimiento más acabado del sistema fónico segmental actual del mapudungún ${ }^{1}$ hablado en Alto Biobío como de la vitalidad fonológica de esta variante hablada en la zona cordillerana 
de la VIII Región de Chile, y, con ello, del impacto del sistema fonéticofonológico segmental del español, en este nivel de la lengua mapuche.

\section{Consideraciones metodológicas}

Los objetivos que nos planteamos con este trabajo fueron: a) determinar las realizaciones con que se manifiestan 12 fonemas del mapudungún hablado en Alto Biobío, que no están presentes en la pauta fonémica del español hablado en $\mathrm{Chile}^{2}$, b) distinguir, entre ellas, las realizaciones indicadoras de vitalidad del sistema fonético-fonológico de la lengua vernácula y las realizaciones indicadoras de transferencias atribuibles al español, c) determinar los porcentajes de realizaciones indicadoras de vitalidad y de transferencia en la macro zona (Alto Biobío), y d) comparar los porcentajes de las realizaciones indicadoras de vitalidad y de transferencias, de acuerdo con las variables género y subsector (valle del río Queuco y valle del río Biobío).

\subsection{Zona}

Para la realización de este estudio, se utilizaron los datos recolectados por los investigadores del proyecto Fondecyt No1131095, Dr. Gastón Salamanca, Dr. Héctor Painequeo Paillán (hablante nativo de mapudungún) y Dr. Jaime Soto. La zona de la cual proceden dichos datos corresponde a la comuna cordillerana de Alto Biobío, ubicada en la VIII Región de Chile. Específicamente, se consideraron las siguientes cinco localidades ubicadas por el sector del valle del río Queuco: Pitril, Cauñicú, Malla-Malla, Trapa-Trapa y Butalelbún; y las siguientes cinco localidades ubicadas por el sector del valle del río Biobío: El Avellano, Chenqueco, Quiñelón, Chevquelavquén y Guallalí-El Barco. Cabe destacar que incluir datos sobre la vitalidad fonético-fonológica segmental del valle del río Biobío constituye un aporte robusto de esta investigación, pues, sobre este punto, no existían reportes anteriores.

Para la descripción del español de Chile, tomamos como referencia el trabajo de Sadowsky y Salamanca (2011). 


\subsection{Participantes e instrumentos de elicitación y registro}

Los participantes fueron 30 colaboradores (3 por cada localidad), los cuales no poseían ningún impedimento físico o mental para realizar la tarea de elicitación requerida. Específicamente, participaron 15 sujetos de género masculino y 15 de género femenino, de entre 20 y 70 años de edad. Previo a los registros, se les solicitó un consentimiento informado a cada uno de ellos.

El instrumento utilizado para la elicitación de los segmentos corresponde a una lista léxica $a d h o c$, que contiene en su mayoría las palabras seleccionadas por Croese (1980). Para las transcripciones, se utilizaron los símbolos propuestos por IPA, en su versión actualizada en 2005. El registro de los datos se realizó con una grabadora TASCAM DR-40 y videocámara digital HANDYCAM SONY DCR-DVD108/DVD308/DVD608/ DVD708. El total de tokens analizados fue de 8800.

\section{Análisis}

\subsection{Con respecto a la cuantificación de las transferencias}

Para realizar el análisis, en primer lugar se determinaron los "fonemas críticos" (Croese, I980 y Henríquez, 2013). Es decir, aquellos segmentos del mapudungún que no son parte del inventario de fonemas del español (de

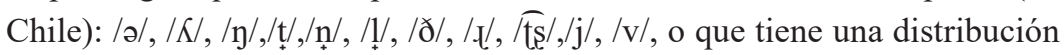
fonotáctica diferente: $/ \mathrm{n} /$ en coda silábica.

A partir de estos fonemas, y luego de la elicitación y transcripción, se identificaron los fonos que se pueden clasificar como "realizaciones indicadoras de vitalidad" del sistema fonético-fonológico del mapudungún (RIV) y "realizaciones indicadoras de transferencias" atribuibles al español (RIT). Estas se presentan en Tabla 1: 
Tabla 1. Realizaciones indicadoras de vitalidad y realizaciones indicadoras de transferencias.

\begin{tabular}{|c|c|c|}
\hline Fonema & $\begin{array}{l}\text { Realizaciones Indicadoras de } \\
\text { Vitalidad (RIV) }\end{array}$ & $\begin{array}{l}\text { Realizaciones Indicadoras de } \\
\text { Transferencias (RIT) }\end{array}$ \\
\hline$/ \mathrm{a} /$ & [ə] [ü] [ə] & {$[\mathrm{i}][\mathrm{e}][\mathrm{a}][\mathrm{o}][\mathrm{u}]$} \\
\hline $\mid K /$ & {$[K][1]$} & 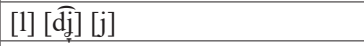 \\
\hline$/ \mathrm{y} /$ & {$[\mathrm{y}]\left[\mathrm{y}^{\mathrm{j}]}[\mathrm{n}][\mathrm{n}][\mathrm{m}][\mathrm{n}]\right.$} & {$[\mathrm{g}]\left[\mathrm{g}^{\mathrm{j}}\right][\mathrm{y}]\left[\mathrm{\gamma}^{\mathrm{j}}\right][\mathrm{b}][\mathrm{n}][\mathrm{j}] \varnothing$} \\
\hline$/ \mathrm{t} /$ & {$[\mathrm{t}][\overline{\mathrm{ts}}][\mathrm{t}]$} & [t] [d] [d] \\
\hline$/ \mathrm{n} /$ & {$[n]$} & {$[\mathrm{n}]$} \\
\hline$/ 1 /$ & {$[1][K][l]$} & [1] [j] \\
\hline$/$ /ठ/ & {$[ð][\theta][0]$} & 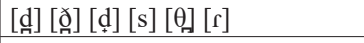 \\
\hline$/ \mathrm{l}$ & {$[-t]\left[x^{y}\right][z] \varnothing$} & {$[\mathrm{c}][\mathrm{dz}][\mathrm{\gamma}]\left[\mathrm{\gamma}^{\mathrm{j}}\right]$} \\
\hline$/ \mathrm{tS}_{\mathrm{S}} /$ & {$[\overline{\mathrm{ts}}][\mathrm{t}]$} & {$[\mathrm{tr}]$} \\
\hline$/ \mathrm{j} /$ & [j] [3] & {$[\widehat{\mathrm{dj}}][\widehat{\mathrm{d}} 3]$} \\
\hline$/ \mathrm{v} /$ & {$[\mathrm{v}][\beta][\mathrm{f}][\phi]$} & {$[\mathrm{b}][\mathrm{b}][\mathrm{p}]$} \\
\hline
\end{tabular}

Finalmente, se determinó el porcentaje total de RIV y de RIT en la macro zona (Alto Biobío), y se comparó el porcentaje de RIV y RIT, de acuerdo con las variables género y subsector (valle del Queuco y valle del Biobío).

\section{Resultados}

\subsection{Porcentajes de realizaciones indicadoras de vitalidad y de transferencias}

En la Figura 1, se presentan los gráficos que contienen los porcentajes de las realizaciones indicadoras de vitalidad y de transferencias para cada uno de los fonemas, y los porcentajes totales.

Figura 1. Porcentajes de realizaciones indicadoras de vitalidad y de transferencias por fonemas, y porcentajes totales.
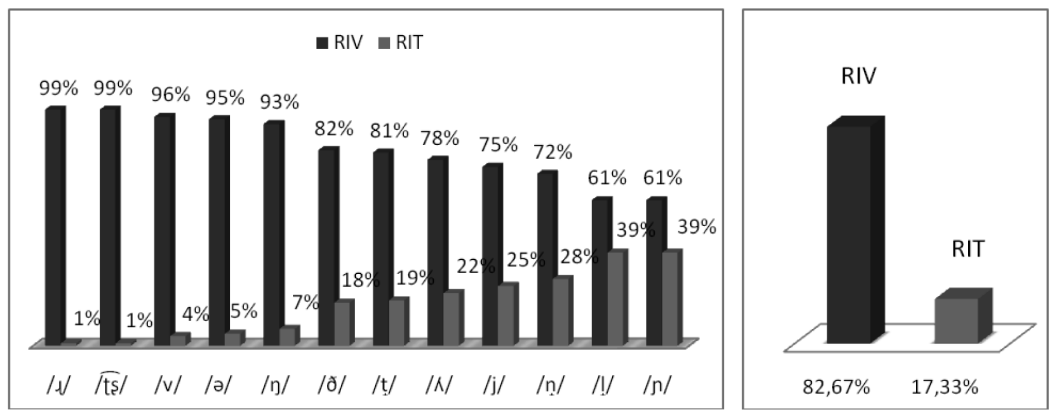

Al observar esta figura,se aprecia que siete de los doce fonemas están en el rango que va del $80 \%$ al $100 \%$ de realizaciones indicadoras de vita- 
lidad. En términos más específicos, cinco de ellos (/. /, / Ts $/, / \mathrm{v} /, / \mathrm{\partial} / \mathrm{y} / \mathrm{y} /)$ están sobre el 90\%, lo que evidencia su importante vitalidad. Destacan particularmente/./ $/ \mathrm{y} / \widehat{\mathrm{T}} /$, por presentar un $99 \%$ de realizaciones vernáculas.

El siguiente grupo está compuesto por los fonemas que se encuentran en el rango que va del $60 \%$ al $80 \%$. Destaca allí la ocurrencia de dos fonemas interdentales. Los porcentajes más bajos corresponden a los fonemas $/ \mathrm{l} / \mathrm{y} / \mathrm{n} /$, ambos con un $61 \%$.

Ahora bien, a pesar de estas diferencias, es importante destacar que todos los fonemas considerados están por sobre el $60 \%$ de realizaciones vernáculas; por lo tanto, en mayor o menor medida, presentan una importante vitalidad.

4.2. Comparación de los porcentajes de realizaciones indicadoras de vitalidad y de transferencias de acuerdo con la variable género

Figura 2. Porcentajes de realizaciones indicadoras de vitalidad y de transferencias en sujetos de género femenino, por fonemas, y porcentajes totales.
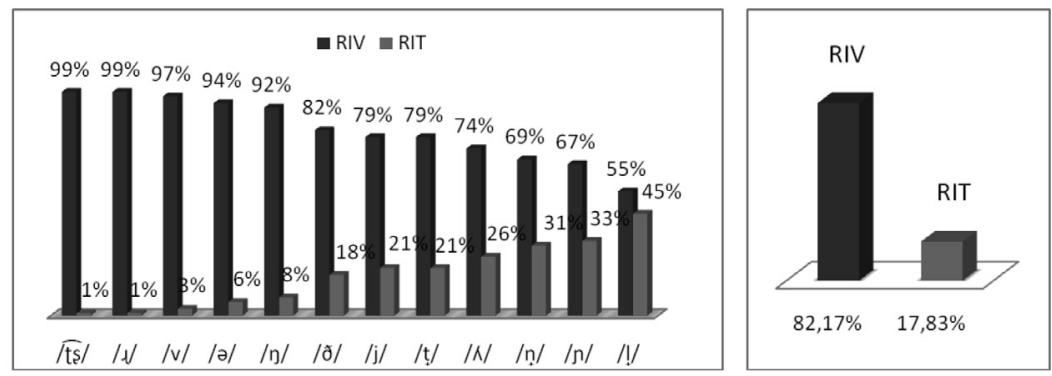

Figura 3. Porcentajes de realizaciones indicadoras de vitalidad y de transferencias en sujetos de género masculino, por fonemas, y porcentajes totales.
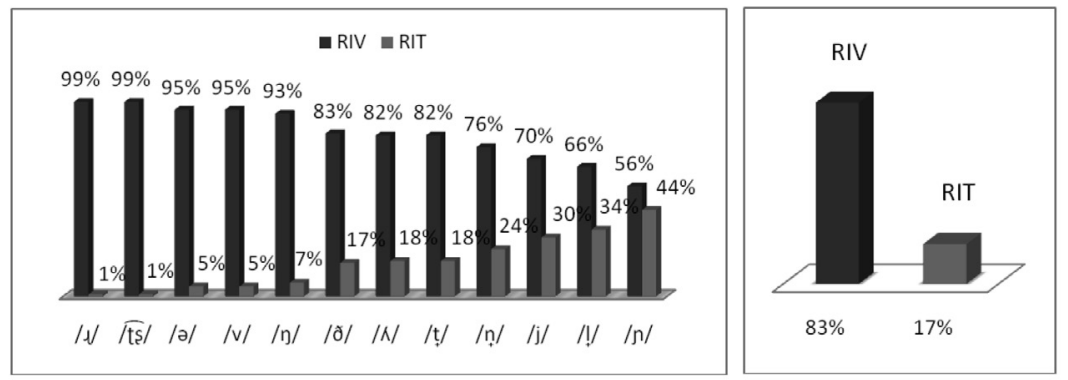
A partir de las figuras 2 y 3, se aprecia que, si consideramos el rango que va del $90 \%$ al $100 \%$, en ambos géneros, ocurren los mismos fonemas que en el conteo general. También, los fonemas $/ \mathrm{t} / \mathrm{y} / \mathrm{t \textrm {S }} / \mathrm{s}$ son los que poseen mayores porcentajes de realizaciones vernáculas. En cuanto a los segmentos que se encuentran entre el $80 \%$ y 90\%, existen ligeras diferencias entre ambos géneros, pues en los sujetos de género femenino sólo el fonema /ð/ ocurre en este rango, mientras que en los sujetos de género masculino, además de este fonema, ocurren $/ \Lambda / \mathrm{y} / \mathrm{t} /$.

Los fonemas que se ubican en el rango que va del 60\% al 80\% de realizaciones vernáculas en el género femenino son: $/ \mathrm{j} /, / \mathrm{t} /, / \Lambda /, / \mathrm{n} / \mathrm{y} / \mathrm{n} /$; y en el género masculino /n/, /j/ y/l/. Nótese, también, la ocurrencia de dos fonemas interdentales dentro de este rango (aunque no son los mismos). Los fonemas con mayor cantidad de transferencias en ambos géneros son/l/ $\mathrm{y} / \mathrm{n} /$, aunque en el orden inverso.

Ahora bien, independiente de las diferencias en los porcentajes de ciertos fonemas, se evidencia una alta homogeneidad entre los sujetos de género femenino y masculino. En efecto, y tal como es posible apreciar en los gráficos que contienen los porcentajes totales, la diferencia es sólo de 0,83 puntos porcentuales.

\subsection{Comparación de los porcentajes de realizaciones indicado-} ras de vitalidad y de transferencias de acuerdo con la variable subsector

A continuación (figuras 4 y 5), se presentan los porcentajes de las realizaciones indicadoras de vitalidad y de transferencias en los subsectores correspondientes a los valles de los ríos Queuco y Biobío, respectivamente.

Figura 4. Porcentajes de realizaciones indicadoras de vitalidad y de transferencias en el valle del río Queuco, por fonemas, y porcentajes totales.
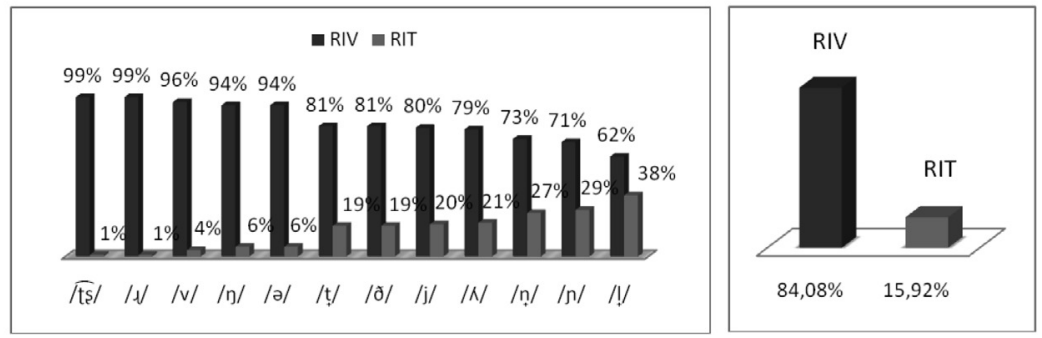
Figura 5. Porcentajes de realizaciones indicadoras de vitalidad y de transferencia en el valle del río Biobío, por fonemas, y porcentajes totales.
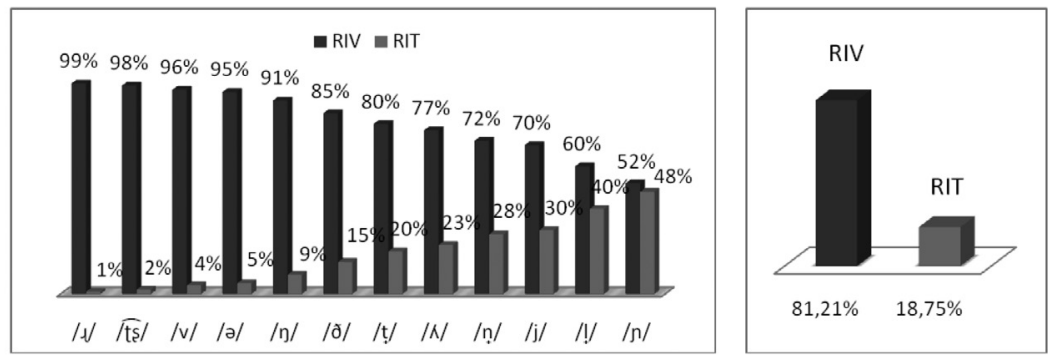

Tal como en el recuento general y de la variable género, los fonemas $/ \mathrm{f} /, / \mathrm{tS} /, / \mathrm{v} /, / \mathrm{o} / \mathrm{y} / \mathrm{y} /$ se incluyen en el rango que va del $90 \%$ al $100 \%$ de realizaciones indicadoras de vitalidad, en los subsectores de Queuco y Biobío. En el rango que va del 80\% al 90\%, ocurren los fonemas /t/ y /ð/, más /j/ en Queuco. En este mismo subsector, los fonemas restantes se ubican en el rango que va desde el $60 \%$ al $80 \%$, siendo /1/ el que presenta menor vitalidad. En cuanto a Biobío, queda fuera de este último rango el fonema /n/, que posee solo un $5 \mathbf{2 \%}$ de realizaciones indicadoras de vitalidad, a diferencia del $71 \%$ que muestra el mismo fonema en Queuco. Nótese también, que, al igual que en el recuento general y de la variable género, en el rango que va del 60\% al $80 \%$ ocurren dos fonemas interdentales, aunque, a diferencia de lo que ocurría en el análisis por género, en el caso de los dos subsectores que nos ocupan, estos fonemas coinciden $(/ \mathrm{n} / \mathrm{y} / \mathrm{l} /)$.

Ahora bien,a pesar de las diferencias que presentan los subsectores de Queuco y Biobío, se puede establecer un comportamiento altamente homogéneo. En efecto, y tal como se observa en el gráfico general, la diferencia es de solo 2.87 puntos porcentuales.

\section{Conclusiones}

En el recuento general, nuestro estudio arrojó un porcentaje total de solo $17,33 \%$ de realizaciones indicadoras de transferencias. Los fonemas que poseen un mayor porcentaje de vitalidad son $/ \mathrm{d} / \mathrm{y} / \mathrm{tS} /$, pues solo un $1 \%$ de sus realizaciones corresponde a realizaciones indicadoras de transferencias. Es importante destacar que nueve de los doce fonemas se encuentran en el rango que va del $75 \%$ al $100 \%$ de realizaciones indicadoras de vitalidad. Al mismo tiempo, destaca el hecho de que en el rango que 
va del $60 \%$ al $75 \%$ de vitalidad se incluyen dos fonemas interdentales: $/ \mathrm{n}$, 1/. Este hecho nos parece interesante, porque estos fonemas poseen una muy baja frecuencia en mapudungún (Lagos, 1981), por lo que, en contacto con segmentos articulatoriamente similares del español, estarían más vulnerables a la pérdida de su vitalidad.

Finalmente, el porcentaje más alto de realizaciones indicadoras de transferencias corresponde a los fonemas /1/ y /n/, con un 38\%. Sin embargo, a pesar de ser los fonemas con mayores porcentajes de transferencias, sus porcentajes no son particularmente elevados.

Con respecto a la variable género, se constató una notable homogeneidad, pues el porcentaje de realizaciones indicadoras de transferencias en el género femenino es de un 17,83\%; y en el género masculino, de un $17 \%$.

Un objetivo importante de esta investigación era conocer la realidad fonético-fonológica de las comunidades ubicadas en el valle del río Biobío, pues, hasta ahora, no se tenía conocimiento de la situación en este subsector. Los resultados muestran una alta vitalidad, pues el porcentaje de realizaciones vernáculas corresponde a un 81,21\%. Caso similar ocurre en el valle del río Queuco, donde un 84,08\% corresponde a realizaciones indicadoras de vitalidad. Así, ambos subsectores también presentan una alta homogeneidad.

Finalmente, reiteramos nuestro agradecimiento a FONDECYT por respaldar este estudio y permitir, con ello, dar respuesta a algunas preguntas relevantes para el estudio del nivel fónico del mapudungún, en general, y del mapudungún (chedungún) hablado en Alto Biobío, en particular. Cabe destacar, en este sentido, que ha sido interesante constatar que en la población adulta de esta zona se mantienen en gran medida las realizaciones de los fonemas que son propias de esta lengua, lo que se distancia de la tendencia presente en una parte importante del territorio donde se habla la lengua mapuche actualmente, con todo lo que ello implica en términos de la vitalidad cultural y lingüística de la "gente de la tierra" ${ }^{3}$. Con todo, será importante observar qué ocurre con la población de menor rango etario de la aquí considerada, pues de esta manera podrá haber un pronunciamiento respecto del grado de transmisión y eventual proyección de la lengua en este nivel.

“Gente de la tierra” es la traducción literal de "mapuche” (de mapu 'tierra' y che 'gente'). 


\section{Referencias bibliográficas}

Álvarez-Santullano, P. (1986). Descripción fonemática del Huilliche: estudio comparativo. Alpha, (2), 45-50.

Appel, R. y Pieter Muysken. (2005). Bilingüismo y contacto de lenguas. Amsterdam: UniversityPress.

Blas Arroyo, J.L. (1991). Problemas teóricos en el estudio de la interferencia lingüística. RSEL (21), 256-289.

Burquest, D. (2009). Phonological Analysis: A functional approach, Dallas. SIL International. Versión electrónica disponible en: http://www. sil. org/silepubs/Pubs/52264/Burquest_ AnalisisFonologico_52264. pdf.

Croese, R. (1980). Estudio dialectológico del mapuche. Estudios Filológicos, (15), 7-38.

Durán, T. y N. Ramos. (1988a). Comportamiento lingüístico en población escolar mapuche contemporánea como expresión del problema de la vitalidad de la lengua. Actas de Lengua y Literatura Mapuche, (3), 57-71.

(1988b). Castellanización formal en la Araucanía a través de la escuela. Lenguas Modernas, (15), 131-154.

Echeverría, M. (1964). Descripción fonológica del mapuche actual. Boletín del Instituto de Filología de la Universidad de Chile XVI, 13-59.

Gundermann, H. et al. (2009). Permanencia y desplazamiento hipótesis acerca de la vitalidad del mapuzugun. $R L A,(47)(1), 37-60$.

Henríquez, M. (2004). Interferencias del sistema fonológico español en el sistema fonológico mapuche de jóvenes hablantes bilingües. RLA, (42), 93-106.

(2013). Vitalidad fonológica del mapudungún en escolares mapuches pewenches y lafkenches de la VIII Región del Bío-Bío. Tesis de Doctorado en Lingüística. Universidad de Concepción.

Henríquez, M. y G. Salamanca. (2015). Vitalidad de la fonología segmental del chedungún hablado por los escolares del Alto Bío-Bío. Alpha, (41), 207-231.

Hernández, A. y N. Ramos. (1978). Rasgos del castellano hablado por escolares mapuches. Estudio de un caso. RLA, (16), 141-149. 
. (1979). Estado actual de la enseñanza del castellano a escolares mapuches del área rural. Un problema de bilingüismo y lenguas en contacto. Estudios Filológicos, (14), 113-127.

(1983). Situación sociolingüística de una familia mapuche. Proyecciones para abordar el problema de la enseñanza del castellano. $R L A,(21), 35-44$.

(1984). Algunas características gramaticales del castellano hablado por mapuches. Actas de Lengua y Literatura Mapuche, (2), 128-138.

Lagos, C. (2012). El mapudungún en Santiago de Chile: vitalidad y representaciones sociales en los mapuches urbanos. $R L A$, (50) (1), 161-184.

Lagos, D. (1981). El estrato fónico del mapudungu(n). Nueva Revista del Pacífico, (19/20), 42-66.

Lagos, D. y S. Olivera. (1988). Algunas características del español hablado por los escolares mapuches de la comuna de Victoria. Estudios Filológicos, (23), 89-102.

Martinet, A. (1984). Elementos de linguistica general. Madrid: Gredos.

Medina López, J. (2002). Lenguas en contacto. Madrid: Arco Libros, S. L.

Mena, D. (2016). Transferencias del español y estatus fonético-fonológico de [S] en el chedungún hablado en Alto Biobío. Tesis de Magíster en Lingüística Aplicada. Universidad de Concepción.

Moreno Fernández, F. (2005). Principios de sociolingüística y sociología del lenguaje, $2^{\circ}$ edición actualizada. Barcelona: Ariel.

Olate, A. y M. Henríquez. (2010). Actitudes lingüísticas de profesores mapuche de Educación Básica: vigencia y enseñanza del mapudungún en el contexto educativo. Literatura y Lingüística, (22), 103-116.

Pike, K. (1961[1947]). Phonemics. A technique for Reducing Languages to writing. The University of Michigan Press: Ann Arbor.

Sadowsky, S. y G. Salamanca. (2011). El inventario fonético del español de Chile: principios orientadores, inventario provisorio de consonantes y sistema de representación (AFI-CL)". Onomázein, (24), 61-84.

Sadowsky, S., H. Painequeo, G. Salamanca y H. Avelino. (2013). Mapudungún. Journal of the International Phonetic Association, 43(1), 87-96. 
Sadowsky, S., M. J. Aninao, M. J. Cayunao y P. Heggarty. (2015). Huilliche: ¿Geolecto del mapudungún o lengua propia? Una mirada desde la fonética y fonología de las consonantes. En Fernández Garay, Ana y María Alejandra Regúnaga (Eds.). Lingüística indigena sudamericana: aspectos descriptivos, comparativos y areales, 23-51. Buenos Aires: FILO:UBA.

Salamanca, G. (1997). Fonología del pehuenche hablado en el Alto BíoBío, $R L A$, (35), 113-124.

Salamanca, G. y E. Quintrileo. (2009). Fonemas segmentales y muestra lexical del mapudungún hablado en Tirúa. $R L A$, (47), 13-35.

Sala, M. (1988). El problema de las lenguas en contacto. México: Universidad Nacional Autónoma de México.

Salas, A. (1971). Problemas de Educación a comunidades mapuches, Primer Seminario de Investigación y Enseñanza de la Lingüística. Actas, acuerdos y recomendaciones. Concepción, Sociedad Chilena de Lingüística y Universidad de Concepción, 105-108.

. (1976). Esbozo fonológico del mapudungún, lengua de los mapuches o araucanos de Chile central. Estudios Filológicos, (11), 143-153. (1983). Alfabetizar y enseñar en mapudungún? ¿Alfabetizar y enseñar en castellano? Alternativas para la escuela rural en la Araucanía chilena. R.L.A, (21), 49-64.

(2006[1992]). El mapuche o araucano, Madrid: Mapfre.

Sánchez, G. (1989). Relatos orales en pewenche chileno. Anales de la Universidad de Chile. Estudios en honor de Yolando Pino Saavedra, 289-360.

Sánchez, M. y G. Salamanca. (2015). El mapuche hablado en Lonquimay: Fonemas segmentales, fonotaxis y comparación con otras variedades. Literatura y Lingüistica, (31), 295-332.

Sepúlveda, G. (1976). Algunos aspectos de la fonología de los préstamos del español al mapudungún. En Tom Dillehay (Ed.). Estudios Antropológicos sobre los mapuches de Chile Sur-Central, 41-68.

Serrano, M. J. (2011). Sociolingüística. Barcelona: Ediciones del Serbal.

Silva-Corvalán, C. (2001). Sociolingüística y pragmática del español. Georgetown: University Press. 
Suárez, J. (1959). The phonemes of an Araucanian dialect. IJAL, (25), 177- 181.

Thomason, S. (2001). Language contact. Edinburgh: University Press Ltd. Trubetzkoy, N. (1987[1939]). Principios de fonología. Madrid: Cincel.

UTEM-CONADI. (2007). Test de competencia Lingüística. Universidad Tecnológica Metropolitana.

Weinreich, U. (1953). Lenguas en Contacto. The Hague, Paris y New York: Mouton.

Wittig, F. (2009). Desplazamiento y vigencia del mapudungún en Chile: un análisis desde el discurso reflexivo de los hablantes urbanos, RLA. (47), 135-155.

Zúñiga, F. (2007). Mapudunguwelaymi am? ¿Acaso ya no hablas mapudungún? Acerca del estado actual de la lengua mapuche. Estudios Públicos, (105), 9-24. 